\title{
Variable Rotation Composite Pulses for High Resolution Nuclear Magnetic Resonance Using Inhomogeneous Magnetic and Radiofrequency Fields
}

\author{
Dimitris Sakellariou, Carlos A. Meriles, \\ Adam Moulé, Alexander Pines* \\ July 8, 2002 \\ Department of Chemistry, UC Berkeley, Berkeley, CA 94720, USA
}

\begin{abstract}
An approach toward high-resolution NMR spectroscopy on samples located outside the physical confines of a magnet (Ex-situ NMR), has recently been described [1]. Nutation echoes are generated by a train of $z$-rotation pulses in the presence of spatially matched static and radio-frequency field gradients. These pulses were based on a combination of perfect $\pi / 2$ constant rotation composite pulses and "imperfect" ordinary pulses of variable length. Here we introduce a new class of "self-compensated" $z$-rotation composite pulses based only on variable rotation inversion pulses that lead to an rf dependent phase shift. Experiments and simulations show that these new pulses perform well at high $B_{0}$ gradients and require less rf power than previous schemes.
\end{abstract}

\section{Introduction}

In recent years a great effort has been devoted to the design of NMR spectrometers with "inside-out" small magnets and surface coils for surface excitation and detection [2-4]. A variety of applications to materials [5,6], well-logging [7], imaging [8] and in vivo NMR [9] have been made possible. However, the large line-broadening caused by the intrinsic 
inhomogeneity of the external magnetic field obscures chemical shift information. Thus, the application of ex-situ NMR has typically been limited to relaxation measurements, imaging and lineshape analysis.

The problem of obtaining high resolution spectra in the presence of field gradients has been addressed previously [10-12], most recently using intermolecular zero-quantum coherences $[13,14]$. Because the net effect of the distant dipolar field is proportional to the static field, it is not practically applicable to the case of inside-out magnets with low magnetic fields $(\leq 2 \mathrm{~T})$.

An approach that makes use of spatially correlated static and radio-frequency fields has recently been demonstrated [1]. Applications to liquids [1], solids, [15] and 2D $J$ homonuclear and heteronuclear correlations in liquids [16] in the presence of inhomogeneous fields have been described. This method is based on the stroboscopic observation of nutation echoes [17] whose amplitudes are modulated by the chemical shift [1]. In this scheme, robust $\pi / 2$ pulses are used in order to direct the magnetization in and out of the $x y$ plane in an ideal manner, and a single ordinary pulse exploits the rf inhomogeneity to encode a spatially dependent phase shift on the spins. Constant rotation $\pi / 2$ composite pulses are typically long and complicated leading to a relatively inefficient pulse train.

Here we describe a new pulse scheme, based on variable rotation composite pulses, to perform $z$-rotations that are proportional to the rf inhomogeneities. Radiofrequency inhomogeneity is exploited throughout the pulse scheme leading to less demanding duty cycles. The performance of these pulses is studied numerically by calculating the NMR spectra in the presence of matched and unmatched field gradients. Experiments performed in model situations within the bore of a superconducting magnet are also presented. It is shown that large field gradients can be refocused and that high resolution NMR spectra can be obtained without any frequency distortions only at the expense of signal amplitude. These observations together with the future possibilities of this method are discussed in 
terms of "real" ex-situ analysis.

\subsection{Z-Rotations and Refocusing sequences}

As mentioned above, this method relies on the spatial matching between the inhomogeneous static $\vec{B}_{0}(\vec{r})$ and the inhomogeneous radiofrequency $\vec{B}_{1}(\vec{r})$ magnetic fields, according to:

$$
\frac{\mathrm{d} B_{0}(\vec{r})}{\mathrm{d} \vec{r}}=k \frac{\mathrm{d} B_{1 \perp}(\vec{r})}{\mathrm{d} \vec{r}}
$$

where the proportionality constant $k$ is not a function of space, and $\vec{B}_{1 \perp}(\vec{r})$ obeys:

$$
\vec{B}_{0}(\vec{r}) \cdot \vec{B}_{1 \perp}(\vec{r})=0
$$

In the following, we treat only the case of correlation along a single dimension, taken to be the longitudinal $x$ axis of the rf-coil.

To obtain a high resolution NMR spectrum, we must refocus the effect of varying Larmor frequencies so that equivalent spins give rise to narrow signals while maintaining chemical shift information. This can be achieved with the aid of composite radiofrequency pulses which have the net effect of a negative sign rotation of the magnetization around the $z$ axis of the rotating frame. This compensates for the evolution of the magnetization under the influence of the inhomogeneous external magnetic field. The rotation of in-plane magnetization about an angle $\beta$ around the $z$-axis can be achieved with the following composite pulse:

$$
P_{z}(\beta)=P_{y}(\pi / 2) R_{x}(\beta) P_{-y}(\pi / 2)
$$

where $R_{\alpha}(\beta)$ and $P_{\alpha}(\beta)$ represent an ordinary square pulse and a composite pulse around the $\alpha$ axis of flip angle $\beta=\omega_{1} \tau_{\beta}$, respectively. In the case of inhomogeneous fields, the $P_{ \pm y}(\pi / 2)$ pulses are constant rotation [18] composite $\pi / 2$ pulses that ideally compensate for rf and static magnetic field inhomogeneities [19], while $R_{x}(\beta)$ should depend only on $\omega_{1}(x)$, since now $\beta=\beta(x)=\left[\omega_{1}+\Delta \omega_{1}(x)\right] \tau_{\beta}$. If we assume that the evolution during this 
last pulse is governed solely by the rf Hamiltonian (strong rf field approximation), $P_{z}(\beta)$ becomes rf dependent, and as a result, a variable phase shift is encoded throughout the sample.

After a time $\tau=\tau_{\beta} \Delta \omega_{1}(x) / \Delta \Omega_{0}(x)=\tau_{\beta} k$, the effect of the inhomogeneous magnetic field is refocused while dephasing induced by chemical shift differences continues to accumulate. Stroboscopic acquisition of the magnetization at the end of each free precession period $\tau$, produces an effective FID with the magnetization after $n$ cycles given by:

$$
M(n \tau)=\sum_{i} I_{i x} \cos \left[n\left(\delta_{i} \tau+\omega_{1} \tau_{\beta}\right)\right]+I_{i y} \sin \left[n\left(\delta_{i} \tau+\omega_{1} \tau_{\beta}\right)\right]
$$

Thus, the spectrum contains chemical shift information $\delta_{i}$. This sequence induces an overall frequency shift of $\omega_{1} \tau_{\beta} / \tau$ which can be corrected either during the sequence by the application of a phase shift at each step or by numerical means while processing the FID.

Although conceptually simple, an effective refocusing pulse train is difficult to implement in practice. Even under the assumptions that the available rf power is strong enough to allow one to disregard offset problems during the pulses and that the rf and static field gradients are perfectly matched, the performance of a pulse like $P_{z}(\beta)$ relies on a combination of ideal $\pi / 2$ pulses and "imperfect" rotations of length $\beta$. At first sight, this does not represent a serious problem given that a variety of composite $\pi / 2$ pulses operating under extended rf and offset inhomogeneities have already been implemented [18]. Most of them operate on a well defined initial condition, i.e. longitudinal magnetization, leading to good excitation or inversion pulses. For our purposes, however, only a reduced subset can be used. We need pulses compensated whose propagator is the wanted rotation operator for any rf field amplitude and offset. This means that their effect is independent on the initial position of the magnetization. This class of pulses, known as constant rotation composite pulses, usually operates over a restricted range of rf values, requires complex phase shifts and are rather long ${ }^{1}$. This last point implies that most of the $\mathrm{rf}$ power is wasted during

\footnotetext{
${ }^{1}$ As a reference, the $z$-rotation pulses used initially were constructed using the constant rotation compos-
} 
the $\pi / 2$ pulses; therefore, the pulse described by Eq. 4 is a rather inefficient refocusing procedure.

In the early 1980's Levitt and Freeman [20], introduced combinations of rf pulses that were used to induce inversion of the $z$ magnetization over a wide range of $\mathrm{rf}$ and/or offset imperfections. Clearly, only $\pi$ rotations about some axis on the equatorial plane can accomplish such a task. This implies that, generally, an effective inversion pulse can be visualized as a series of $\pi$ rotations taking place around a wide distribution of axes on the $x y$ plane. If the directions of these axes depend on the rf, a spatially variable phase shift can be attained when an initial $\pi$ pulse is concatenated with a second inversion displaying a new set of axes. One can combine two of these $\pi$ pulses to form a $2 \pi$ composite pulse that introduces a net $z$ phase shift. As an example of this approach, we consider the first $\pi$ composite pulse:

$$
R_{x}(\pi / 2) R_{y}(\pi) R_{x}(\pi / 2)
$$

which leads to:

$$
P_{z}=R_{x}(\pi / 2) R_{y}(\pi) R_{x}(\pi / 2) R_{x}(\pi / 2) R_{-y}(\pi) R_{x}(\pi / 2)
$$

When the rf field is inhomogeneous, a given deviation $\epsilon$ from the nominal angle leads to the altered $\pi$ rotation:

$$
R_{x}(\pi / 2+\epsilon) R_{ \pm y}(\pi+2 \epsilon) R_{x}(\pi / 2+\epsilon)
$$

which, to first order, can be rewritten as [20]:

$$
R_{\mp z}(\epsilon) R_{y}(\pi) R_{ \pm z}(\epsilon)
$$

This result reveals that if the rf variation is small enough $(\epsilon<1)$, the pulse behaves as a $\pi$ rotation about an axis shifted from the nominal one by an amount proportional to this ite $\pi / 2$ pulse introduced by Wimperis [19]. Using this composite pulse Eq. 3 becomes: $P_{z}(\beta)=R_{97.2}(\pi)-$ $R_{291.5}(2 \pi)-R_{97.2}(\pi)-R_{0}(\pi)-R_{x}(\beta)-R_{277.2}(\pi)-R_{111.5}(2 \pi)-R_{277.2}(\pi)-R_{180}(\pi)$ 
variation. The sign of the phase shift depends on the relative phases within the composite $\pi$ pulse. Combining expressions 6 and 8 , pulse $P_{z}$ can be expressed to first order as :

$$
P_{z}=R_{-z}(4 \epsilon)+O\left(\epsilon^{2}\right)
$$

This pulse can be used to create a variable phase shift on spins subject to an inhomogeneous rf field. Fig. 1(a) illustrates the trajectory of the magnetization for $\pm 20 \%$ rf inhomogeneity. The final position of the magnetization lies on the $x y$ plane, dephased by an rf dependent phase shift. If the shape of the field gradient is matched, dephased spins can be refocused when free evolution occurs on a time interval of proper length. For stronger gradients, repetition of the same pulse unit two times or more allows the retention of a reasonably long free evolution period during the pulse train. A schematic of the refocusing pulse train is shown in Fig. 1(b): block $Q$ contains, potentially, any combination of $P_{z}$ composite pulses whose relative phases can be shifted to correct for higher order artifacts in the spectrum.

\subsection{Experiments and Simulations}

Experiments were performed in an ultra-widebore imaging magnet using a Chemagnetics Infinity NMR spectrometer operating at a proton frequency of $179.12 \mathrm{MHz}$ and a homebuilt imaging probehead with three perpendicular gradient coils. The excitation coil has a conical shape and was designed to generate a linear radiofrequency gradient along the $x$ axis as shown in Fig. 2. Numerical integration of the Biot-Savart law gives the field contour levels shown in Fig. 2(b) which display a linear dependance in the sample region. To minimize imperfect spatial matching, an extended $(1.2 \mathrm{~cm}$ long) but narrow (1 mm diameter) tube containing ethanol was placed inside the coil. This configuration produces a nominal value of the rf amplitude of $15 \mathrm{kHz}$ for protons, measured as the optimal $\pi / 2$ pulse length, in the absence of any $B_{0}$ gradients. The intrinsic static field homogeneity can be estimated from Fig. $3(\mathrm{a})$ to be $\approx 0.5 \mathrm{ppm}$. A variable gradient $\mathrm{d} B_{0} / \mathrm{d} x$ of 6.5 to 50 
$\mathrm{kHz} / \mathrm{cm}$ along the $x$ axis was applied in the following experiments. As shown in Fig. 3(b), the variation in Larmor frequencies ranges from 40 to $350 \mathrm{ppm}$ (7 to $63 \mathrm{kHz}$ ), and always obscures the chemical shift. Well resolved spectra are obtained using the pulse sequence of Fig. 1(b) and are presented in Fig. 3(c). In all spectra reported within this paper 16 scans were acquired. As the gradient increases, a loss in signal to noise ratio is observed without significantly affecting the spectral resolution.

It is important to note that the limiting $B_{0}$ gradient that can be addressed by this methodology scales with the amplitude of the rf field. To illustrate this, numerical simulations were performed using larger rf amplitudes and field gradients. An ensemble of 200 "molecules" (1200 spins 1/2) was prepared in a one-dimensional arrangement under constant linear $B_{0}$ and $\mathrm{rf}$ gradients. The latter is set such that the last molecule in the chain does not experience any rf. The spectrum is composed of three distinct lines encompassing a $6 \mathrm{ppm}$ spread and having a linewidth of $0.3 \mathrm{ppm}$. This linewidth is introduced by a phenomenological $T_{2}(5 \mathrm{~ms})$ damping in the FID. The effect of progressively higher field gradients pointing along the chain is displayed in Fig. 3(d). The calculated response when the refocusing pulse train is applied is shown in Fig. 3(e). Note that a nominal rf amplitude of $83 \mathrm{kHz}$ was used. The chemical shift information is left unaltered even in the presence of very high gradients $(\approx 170 \mathrm{kHz} / \mathrm{cm})$. These values are comparable with those found in "inside-out" magnets [2,7], and suggest that such schemes could give high resolution ex-situ NMR spectra provided that field and rf gradients are properly matched.

In accordance with the experiments, Fig. 3(e) also shows a reduction of the refocused signal as the gradient increases. The reason for this behavior becomes apparent from Fig. 4, where the contributions from three different parts of the chain to the overall spectrum have been simulated and compared to those expected in the absence of static field inhomogeneity. In accordance with the reciprocity theorem [21], the largest contribution comes, in both cases, from the first section (first quarter) which encounters the strongest rf amplitude. It 
is critical to note that, in case (b) the carrier frequency was set close to resonance for spins located at the first quarter of the sample; this implies that the performance of the pulse train is optimum in this region. However, a proper setting of the carrier frequency can, in principle, be utilized to obtain spectra from other portions of the sample at the expense of sensitivity.

The perfect matching assumed to hold between the rf and static field gradient must be stressed; if this were not the case, the performance of the pulse sequence would invariably be reduced. In the case of mismatch along the sample axis, the localized behavior of the pulse train can be utilized, and the influence of the mismatch can be minimized. However, mismatching along a plane perpendicular to the sample axis leads to imperfect refocusing because the spins cannot be spatially discriminated by utilizing the one-dimensional rf inhomogeneity. Fig. 5 shows the effect of a slight mismatch ( $1 \%$ of the nominal rf value) between the $\mathrm{rf}$ and $B_{0}$ gradients along this axis on the spectum. In panel (a) a relatively small $B_{0}$ gradient $(25 \mathrm{kHz} / \mathrm{cm})$ is refocused under perfect (left) and imperfect (right) matching. The effect of the mismatch is not detrimental to the resolution of the spectrum. This seems not to be true when large $B_{0}$ gradients $(170 \mathrm{kHz} / \mathrm{cm})$ are refocused, as in panel (b). Extensive use of rf-inhomogeneity renders the pulse sensitive to imperfections due to matching: accumulated refocusing errors compromise the spectral resolution.

\subsection{Extensions}

In the previous sections, it was shown that pulses that perform non-ideally throughout the sequence are combined so that their overall effect shows a dependance on the desired physical parameter ( $\mathrm{rf}$ inhomogeneity). Using this method, some constraints in the development of new pulse schemes are "relaxed"; all spins do not have to behave identically. The description of a $z$-rotation pulse as a combination of variable rotation inversion pulses, allows one the opportunity to implement a set of compensated pulses having varying features. As 
demonstrated by Levitt and Freeman [22], a rotation of the form $R(\pi / 2)_{x}-R(\gamma)_{y}-R(\pi / 2)_{x}$ can be made offset insensitive to first order if $\gamma$ is chosen to be close to $3 \pi / 2$. In the present context, a z-rotation pulse can then be envisaged having the form :

$$
R_{x}(\pi / 2) R_{y}(3 \pi / 2) R_{x}(\pi) R_{-y}(3 \pi / 2) R_{x}(\pi / 2)
$$

It is interesting to note that there is no obvious method by which to make such compensation for a pulse having the form of Eq. 3. Even if the $\pi / 2$ pulses are chosen to be robust with respect to offset, the only manner to compensate the $\beta$ flip angle pulse for offsets is to increase the rf amplitude. As a second possibility, the extent of the rf bandwidth can be improved [23] by replacing the $\pi / 2$ in Eq. 5 by $\pi$ pulses. This gives the following $z$-rotation pulse:

$$
R_{x}(\pi) R_{y}(\pi) R_{x}(2 \pi) R_{-y}(\pi) R_{x}(\pi)
$$

whose performance could be further extended by applying iterative schemes on the inversion pulses [23].

In general, the phase shift encoded by a series of pulses (like those of Eq. 10,11) is not a linear function of the rf and, therefore, it is the combined effect of the $B_{1}$ gradient and the pulse response to the rf that determines the phase shift encoded at each portion of the sample. In principle, this represents an extra degree of freedom because an effective rf profile could be crafted by selecting pulses such that an optimized matching takes place for a given rf-offset correlation band.

\subsection{Conclusions}

Recent advances toward high-resolution ex-situ NMR spectroscopy require the development of new compensated pulse sequences for refocusing. In this paper we have introduced a novel method to construct $z$-rotation pulses that can be used to recover chemical shift information in the presence of spatially matched $\mathrm{rf}$ and $B_{0}$ fields. This procedure has been 
shown to be efficient in the presence of the high gradients expected to be present in real ex-situ experiments. The sensitivity of the pulse to carrier frequency offset renders its performance localized, leading to a loss of signal. However, the spectral resolution is not affected, and different parts of the sample can be analyzed by properly tuning the carrier frequency. In a real ex-situ situation, the gradient profiles for the rf and $B_{0}$ fields are not necesarily linearly correlated; but imperfect matching along the direction of the field gradient does not detrimentally affect the spectrum due to the intrinsic localization of the pulse. This is not true for mismatching perpendicular to the excitation axis where other means of spin discrimination are needed. The use of a system composed of two coils of different size could be a potential solution to this problem if, of all the spins excited, only those located in a well matched region are detected by a smaller pick-up coil. Finally, it is important to mention that because the inhomogeneous broadening on samples located outside of the magnet greatly exceeds the rf amplitude, the use of any pulse scheme, such as those discussed here, will invariably lead to a loss in sensitivity. This is due to the fact that only those spins whose frequency differs by less than the rf amplitude can be controlled simultaneously for a given tuning frequency. To manipulate a larger portion of the sample, adiabatic pulses that are able to spatially discriminate between spins are desired. Work in this direction is currently underway in our laboratory.

\subsection{Acknowledgments}

This work was supported by the Director, Office of Science, Office of Basic Energy Sciences, Materials Sciences Division, of the U.S. Department of Energy under Contract No. DEAC03-76SF00098. 


\section{References}

[1] C. A. Meriles, D. Sakellariou, H. Heise, A. J. Moulé and A. Pines, Science 293 (2001) 82.

[2] G. Eidmann, R. Savelsberg, P. Blümler and Blümich, J. Magn. Reson. A 122 (1996) 104.

[3] B. Blümich, P. Blümler, G. Eidmann, R. Haken, U. Schmitz, K. Saito and G. Zimmer, Magn. Reson. Imag. 16 (1998) 479.

[4] F. Bãlibanu, K. Hailu, D. E. Demco and B. Blümich, J. Magn. Reson. 145 (2000) 246.

[5] A. Guthausen, G. Zimmer, P. Blümler and B. Blümich, J. Magn. Reson. 130 (1998) 1.

[6] G. Zimmer, A. Guthausen and B. Blümich, Solid State Nuclear Magn. Reson. 12 (1998) 183.

[7] R. L. Kleinberg, A. Sezginer and D. D. Griffin, J. Magn. Reson. 97 (1992) 466.

[8] J. Prado, B. Blümich and U. Schmitz, J. Magn. Reson. 144 (2000) 200.

[9] R. Haken and B. Blümich, J. Magn. Reson. 144 (2000) 195.

[10] D. P. Weitekamp, J. R. Garbow, J. B. Murdoch and A. Pines, J. Am. Chem. Soc. 103 (1981) 3578 .

[11] M. Gochin, D. P. Weitekamp and A. Pines, J. Magn. Reson. 63 (1985) 431.

[12] J. J. Balbach, M. S. Conradi, D. P. Cistola, C. Tang, J. R. Garbow and W. C. Hutton, Chem. Phys. Lett. 277 (1997) 367. 
[13] W. S. Warren, W. Richter, A. H. Andreotti and B. T. Farmer II, Science 262 (1993) 2005.

[14] S. Lee, W. Richter, S. Vathyam and W. S. Warren, J. Chem. Phys. 105 (1996) 874.

[15] C. A. Meriles, D. Sakellariou and A. Pines, Chem. Phys. Lett. 358 (2002) 391.

[16] H. Heise, D. Sakellariou, C. A. Meriles and A. Pines, J. Magn. Reson. 156 (2002) 146.

[17] I. Ardelean, A. Scharfenecker and R. Kimmich, J. Magn. Reson. 144 (2000) 45.

[18] M. H. Levitt, The encyclopedia of NMR (J. Wiley \& Sons, London, 1997).

[19] S. Wimperis, J. Magn. Reson. A 109 (1994) 221.

[20] M. H. Levitt and R. Freeman, J. Magn. Reson. 33 (1979) 473.

[21] D. I. Hoult and R. E. Richards, J. Magn. Reson. 24 (1976) 71.

[22] R. Freeman, S. P. Kempsell and M. H. Levitt, J. Magn. Reson. 38 (1980) 453.

[23] A. J. Shaka and R. Freeman, J. Magn. Reson. 59 (1984) 169. 
Fig. 1: (a) Trajectory of the transverse magnetization during the $z$-rotation composite pulse of Eq. 6. In the left and the right sphere the dephasing is introduced by $\pm 20 \% \mathrm{rf}$ inhomogeneity, respectively, with the initial magnetization along the $x$ axis. If the field has the nominal rf amplitude a perfect $2 \pi$ pulse is obtained, if it has greater (less) than the nominal value a positive (negative) phase shift is induced. (b) Pulse sequence used for the detection of chemical shift in the presence of inhomogeneous $B_{0}$ magnetic field. After an initial $\pi / 2$, pulse a variable phase shift is created by means of the pulse series $Q$ which is composed of an integral number of $z$ rotation $P_{z}$ pulses. The dwell time is set such that a nutation echo is formed at the end of it. The amplitude of the echo is modulated with the chemical shift. Thus, stroboscopic detection for the refocused echo points yields an inhomogeneity free FID.

Fig. 2: Schematic design of the rf coil used for excitation and detection. A conical coil was used to obtain a linear gradient of the rf field within the cavity. All dimensions are in mm. The contour plot, obtained after exact integration of the Biot-Savart law assuming zero wire thickness, shows the theoretical profile of the rf field.

Fig. 3: (a) Standard one pulse spectrum of pure ethanol. (b) Standard one pulse spectra in the presence of a linear $B_{0}$ field gradient. (c) Spectra obtained in the presence of the respective gradient using the sequence of Fig.1. (d) and (e) are the simulated equivalents for (b) and (c). The nominal value of the rf field was set to $83 \mathrm{kHz}$ for the spins located closest to the coil. A linear rf gradient was applied so that the rf amplitude for the spins located the furthest from the coil experience zero rf amplitude. The basic blocks $P \bar{P}$ for $G_{0}=25 \mathrm{kHz} / \mathrm{cm}, P \bar{P} \bar{P} P$ for $G_{0}=45$ and $60 \mathrm{kHz} / \mathrm{cm}$, and $P \bar{P} \bar{P} P P \bar{P} \bar{P} P$ for $G_{0}=87$ and $G_{0}=170 \mathrm{kHz} / \mathrm{cm}$ were used. 
Fig. 4: Contributions from three different regions of the sample. The spectra are simulated as in Fig. 3(e) using $G_{0}=25 \mathrm{kHz} / \mathrm{cm}$. The first quarter of the sample, closest to the coil gives a sensitive resolved spectrum because the carrier frequency was deliberately chosen to be nearly on resonance for those spins. The second quarter contributes less to the total FID and suffers more from imperfect refocusing. The remainder of the sample does not contribute significantly to the recorded spectrum. This figure clearly illustrates that the performance of the pulse train is localized and that proper adjustment of the carrier frequency could lead to localized spectra at the expense of sensitivity.

Fig. 5: Refocused spectra in the presence of perfect (left panels) and imperfect (right panels) rf and static field matching. The refocused gradient was $G_{0}=25 \mathrm{kHz} / \mathrm{cm}$ for the (a) panels and $G_{0}=170 \mathrm{kHz} / \mathrm{cm}$ for the (b) panels. The linear correlation along the $x$ axis, used for the perfect matching, was adversely affected by the addition of a rf gradient along the $y$ axis. This extra perturbation was only of $\% 1$ the main $\mathrm{rf}$ field which was $83 \mathrm{kHz}$ at the portion of the sample nearest the coil. We observe that this slight mismatch is not vital for the refocusing when the gradient is small, but it can have detrimental results for high $B_{0}$ gradients. 
Variable Rotation Composite Pulses for High Resolution Nuclear Magnetic Resonance Using Inhomogeneous Magnetic and Radiofrequency Fields

Sakellariou et. al.

Fig. 1

(a)
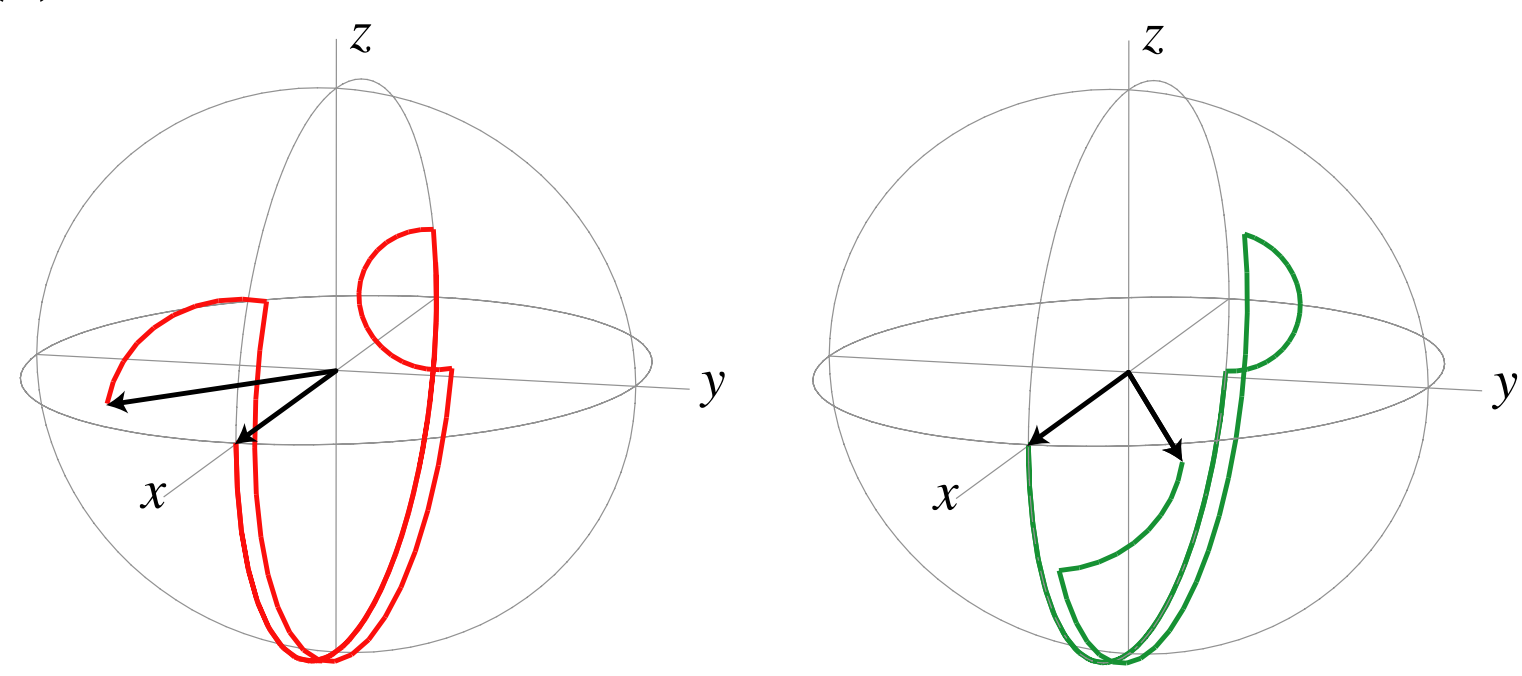

(b)

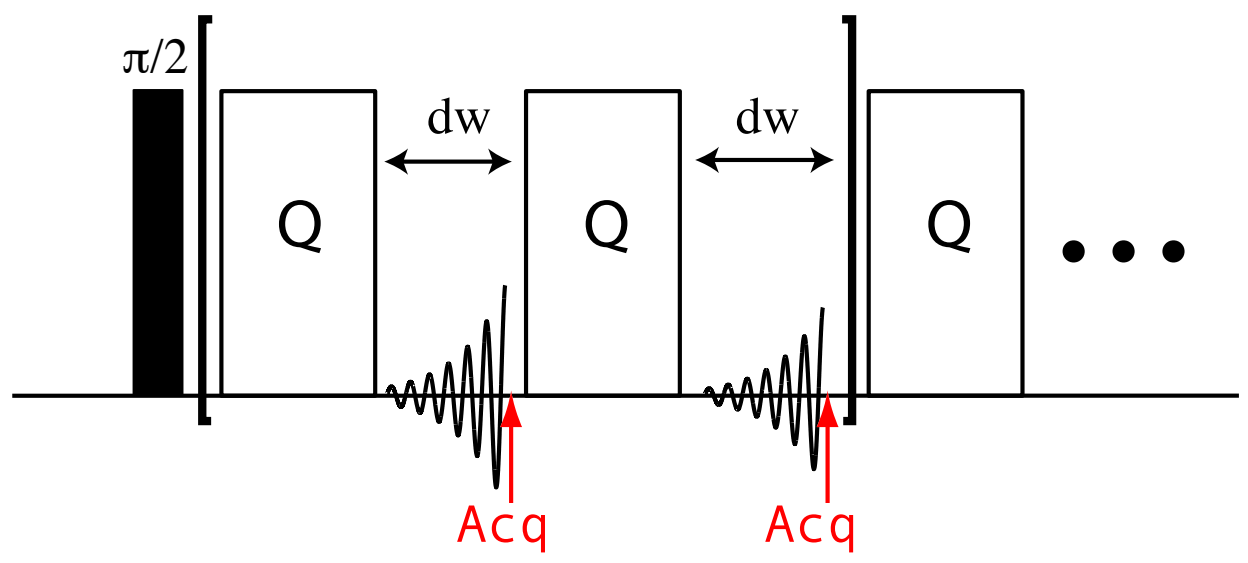


Variable Rotation Composite Pulses for High Resolution Nuclear Magnetic Resonance Using Inhomogeneous Magnetic and Radiofrequency Fields

Sakellariou et. al.

Fig. 2
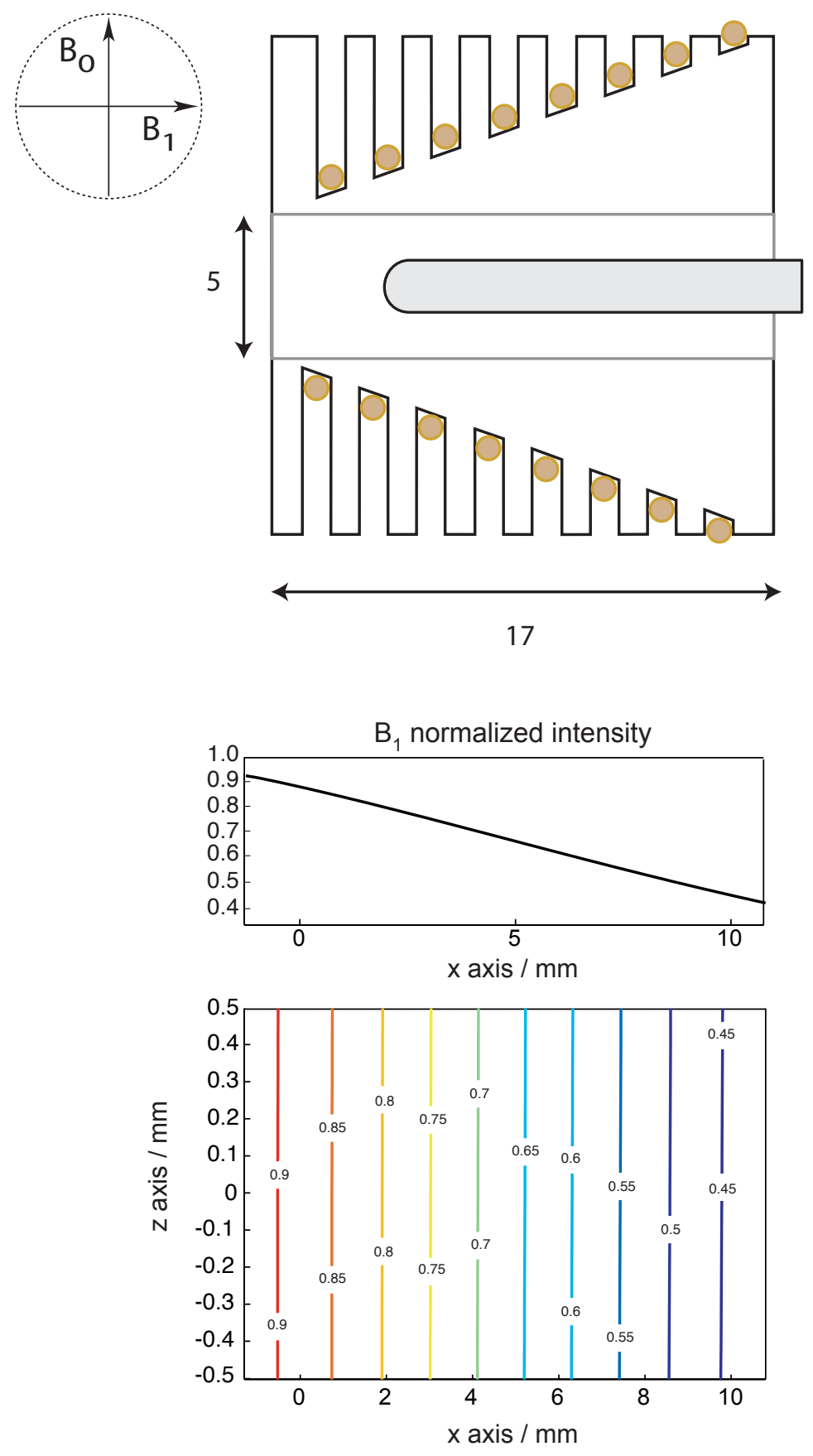
Variable Rotation Composite Pulses for High Resolution Nuclear Magnetic Resonance Using Inhomogeneous Magnetic and Radiofrequency Fields

Sakellariou et. al.

Fig. 3

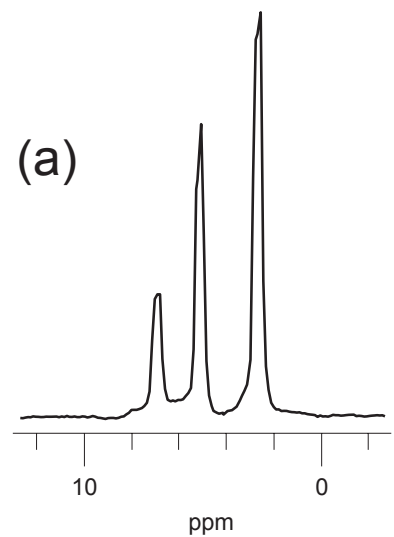

(b)

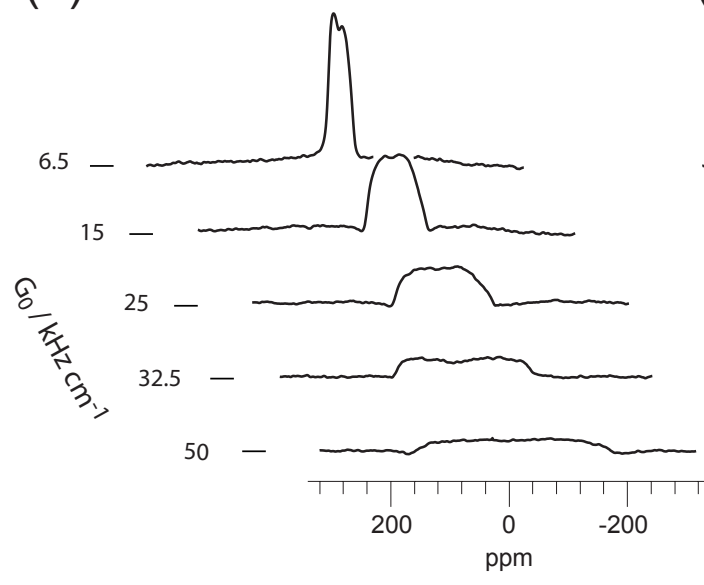

(d)

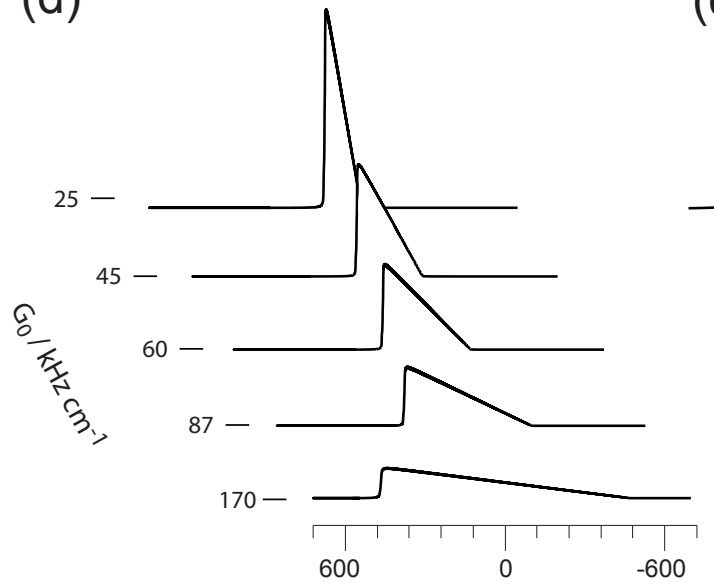

(c)

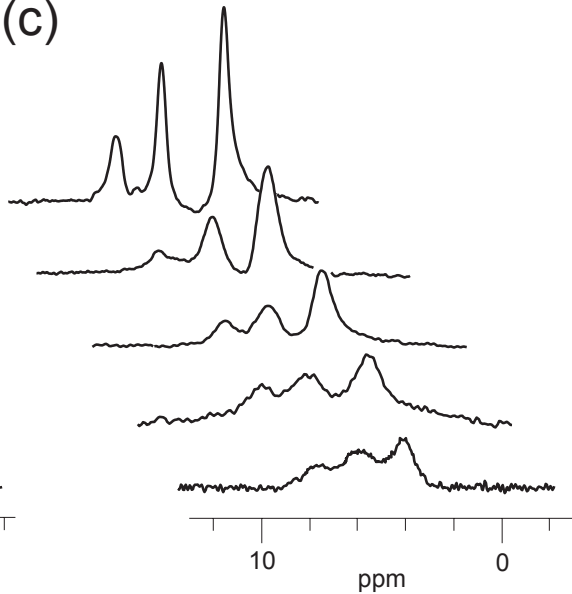

(e)

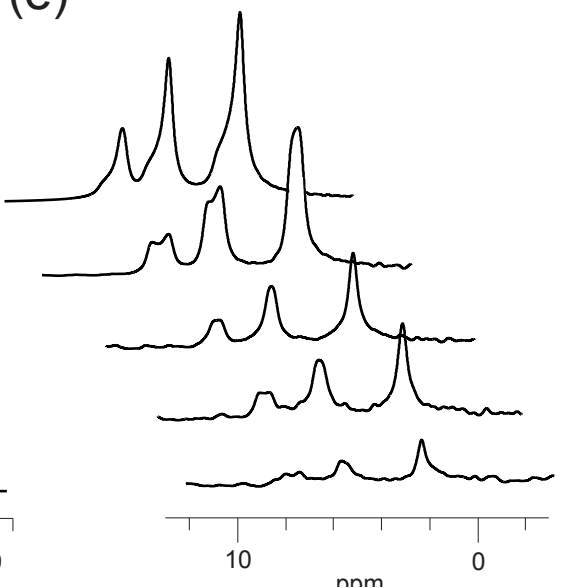


Variable Rotation Composite Pulses for High Resolution Nuclear Magnetic Resonance Using Inhomogeneous Magnetic and Radiofrequency Fields

Sakellariou et. al.

Fig. 4
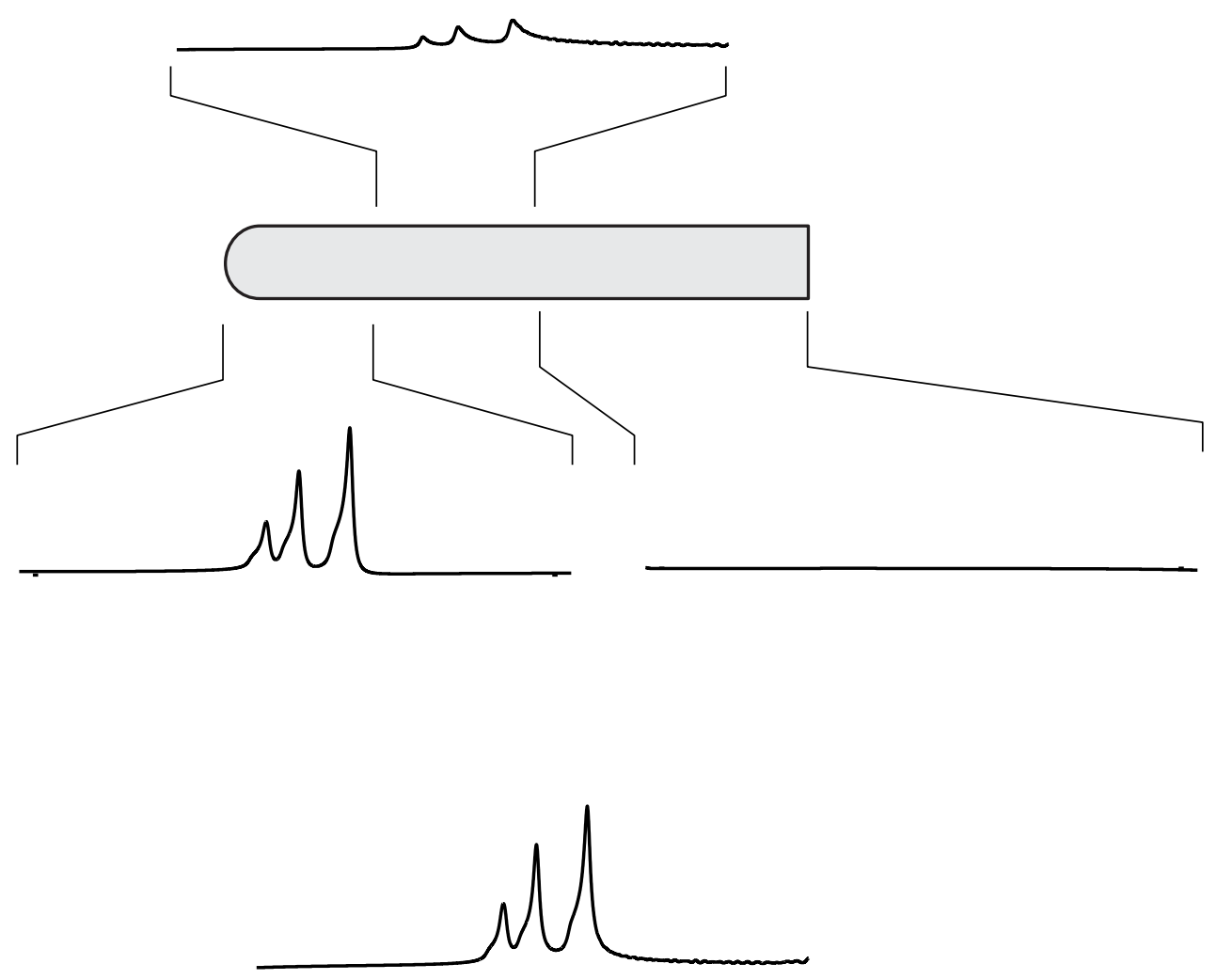

Total 
Variable Rotation Composite Pulses for High Resolution Nuclear Magnetic Resonance Using Inhomogeneous Magnetic and Radiofrequency Fields

Sakellariou et. al.

Fig. 5

(a)
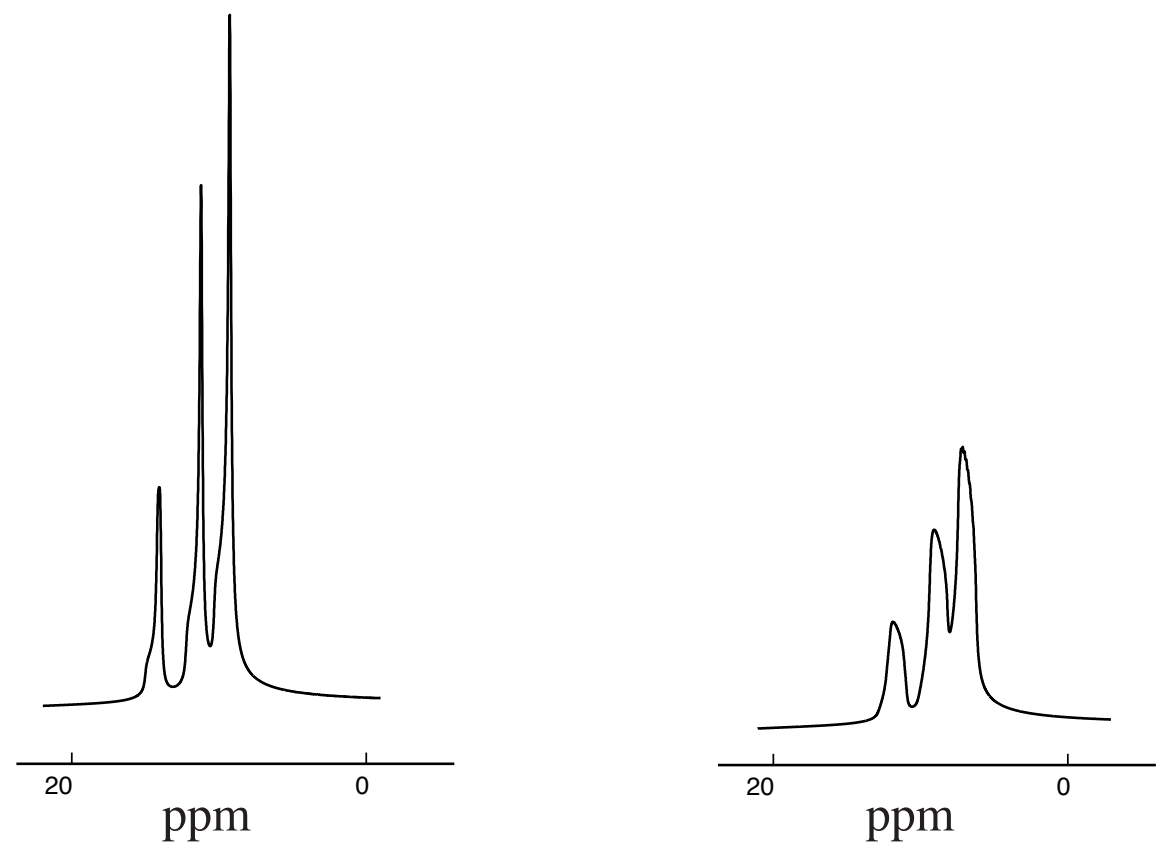

(b)
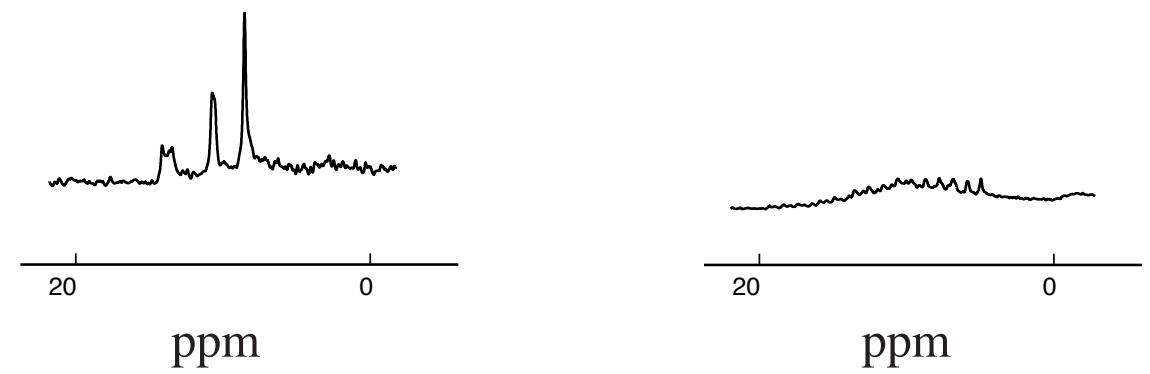